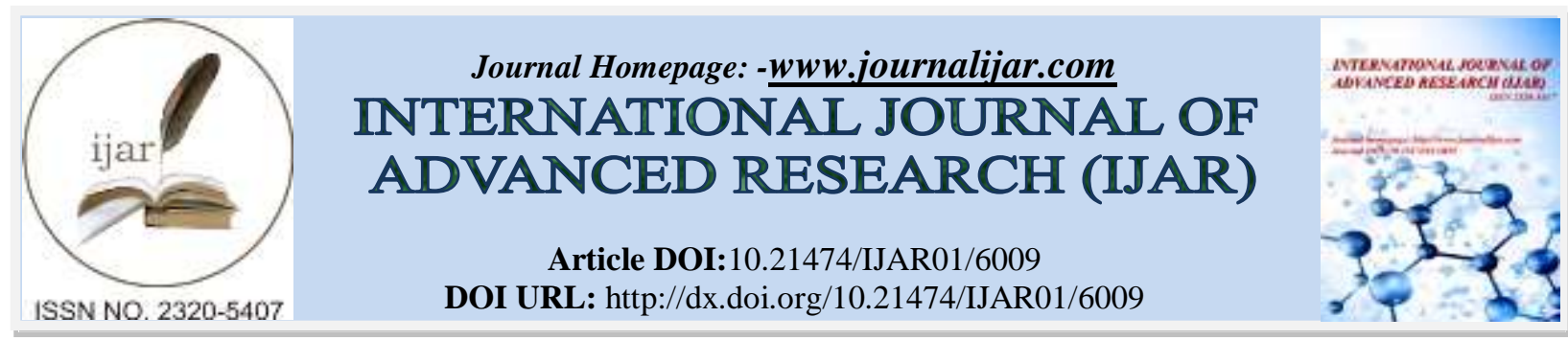

RESEARCH ARTICLE

\title{
ACCURACY OF ANORECTAL MANOMETRY IN THE DIAGNOSIS OF HIRSCHSPRUNG'S DISEASE IN CHILDREN.
}

Dr. Zaffer Saleem Khanday, Dr. NaseerAwan and Prof. RekhaPatnaik.

Department of Pediatric Surgery, Sher-i-Kashmir Institute of Medical Sciences, Soura, Srinagar, Kashmir.

\section{Manuscript Info}

Manuscript History

Received: 08 October 2017

Final Accepted: 10 November 2017

Published: December 2017

Key words:-

Anorectalmanometry, Hirschsprung's disease.

\section{Abstract}

Aim: Accuracy of Anorectal Manometry in the Diagnosis of Hirschsprung's Disease in Children

Materials and Methods: This was a case control study conducted in the Department of Paediatric Surgery, Sher-I-Kashmir Institute of Medical Sciences from December 99 to June 2002.Control subjects consisted of 50 children whose ages ranged from 1 day to 13 years. None of the controls had any history of defecation disorder. All the controls had rectoanal inhibitory reflex present at the time of examination. The patient group consisted of 50 children with constipation.

Results: All the controls had rectoanal inhibitory reflex present at the time of examination. Resting rectal pressures recorded in them ranged from 5-11 cmH,0 and the Mean anal canal pressure ranged from 14-54 $\mathrm{cm}$ H2O.Functional anal canal length ranged from $1.0-3.5 \mathrm{cms}$.In our series $50 \%$ of patients were diagnosed to be suffering from Hirschsprung's disease and $33.3 \%$ were diagnosed to be suffering from idiopathic constipation. Youngest patient was 10 days old who presented with necrotizing enterocolitis of Hirschsprung's disease and the oldest presented as a case of neglected chronic constipation in intestinal obstruction. Anorectal pressure profile did not differ much between the control group and the patient group with average resting rectal pressure ranging from $4-14 \mathrm{cmH} 20$ and mean anal canal pressure ranging from 10-48 $\mathrm{cmH} 20$. The length of the anal canal averaged 1.0$3.0 \mathrm{cms}$. There were 3 false negatives and 2 false positives for Hirschsprung's disease on anorectalmanometry with overall diagnostic accuracy of $83.3 \%$.

Conclusion: Anorectal Manometry is an accurate, noninvasive test which has a place as screening test of choice for Hirschsprung's disease.

Copy Right, IJAR, 2017,. All rights reserved.

\section{Introduction:-}

Congenital megacolon or Hirschsprung's disease is one of the classic problems in pediatric surgical practice. It was Harold Hirschsprung's a Danish Pediatrician, who first described it as a distinct entity. His two patients died at 8 and 11 months respectively, with unrelenting constipation, malnutrition and enterocolitis. He presented these cases at Berlin in 1886 and emphasized on the postmortem findings of colonic distension and muscular hypertrophy proximal to a smaller

Corresponding Author:-Zaffer SaleemKhanday.

Address:-Department of Surgerv Govt Medical College.Karannager. Srinagar. Kashmir.190005 
normal sized rectum. But that time megacolon was thought to be the offending entity and the surgeons tried to excise the dilated segment, but achieved no success. In 1945 Neuhauser's radiologic observation of a smaller than normal rectum, transition zone to a megacolon and normal contractions in the left colon that did not continue into the recto sigmoid and pathologic findings of aganglionosis in patients with same clinical picture published synchronously by Zuelzer and Wilson in 1948 completed the clinical correlation as Hirschsprung's disease.(1)

\section{Patho physiology:-}

The basic path physiology in Hirschsprung's disease is a lack of propagation of propulsive waves or an abnormal or absent relaxation of the internal anal sphincter due to aganglionosis, hypoganglionosis or dysganglionosis of the bowel. Aganglionic bowel has an increased number of both cholinergic and adrenergic nerves, a normal tissue content of acetylcholine but elevated release in response to stimulation, an elevated acetylcholine esterase concentration and elevated tissue catecholamine and a decrease in certain peripheral nerve fibers (1)

\section{Diagnosis:-}

Once the diagnosis of aganglionosis is entertained from clinical findings, several confirmatory steps exist, including radiologic studies, anorectalmanometry, and in particular, histological examination of the rectal wall biopsy specimens.

\section{Radiologic Diagnosis:-}

Plain abdominal films in a neonate will show dilated loops of bowel with fluid levels. Pneumoperitonium may be found in those with perforation. A barium enema performed by careful technique should achieve a high degree of reliability in diagnosing Hirschsprung's disease in the newborn. A typical case of Hirschsprung's disease demonstrates the flow of barium from the undilated rectum through a cone - shaped transitional zone into the dilated colon. A delayed film at 24 hours may confirm the diagnosis by demonstrating the retained barium and often accentuating the appearance of the transitional zone.

\section{Anorectal Manometry:-}

Anorectal Manometry has been evaluated as a diagnostic modality in Hirschsprung's disease. Hirschsprung's disease is characterized by high pressure, progressive peristalsis in the proximal ganglionic bowel, lack of progressive peristalsis in a normal pressure zone lacking ganglion cells, and failure of relaxation of the rectal sphincter in response to rectal distention. In normal person, when the rectal balloon is distended with air, the rectum immediately responds with a transient rise in pressure and at the same time the internal sphincter's rhythmic activity is depressed or abolished and its pressure falls. (2)

A normal rectoanal response indicated that Hirschsprung's disease could be safely ruled out as the cause of abdominal distention and constipation. Anorectalmanometry is important when there is ambiguity of histology or the child is too sick to be subjected to traumatic procedures.

\section{Rectal biopsy:-}

Rectal biopsy is the diagnostic standard for Hirschsprung's disease. The biopsy must be taken 2 to $3 \mathrm{~cm}$ proximal to the pectinate line because of normal physiologic hypoganglionosis in the most distal rectum. Adequacy is determined by evidence of sufficient tissue to demonstrate hypertrophic nerves associated with the absence of ganglion cells. Full thickness rectal biopsy remains the gold standard because the specimen allows evaluation of Auerbach's intermyenteric plexus as well as Meissner'ssubmucosal plexus and Henle's or the deep submucosal plexus. (3)

\section{Materials and Method:-}

The study of AnorectalManometry in the diagnosis of Hirschsprung's disease was conducted in the Department of Paediatric Surgery, Sher-I-Kashmir Institute of Medical sciences, Soura, Srinagar. The study was undertaken on healthy control group comprising of 50 normal children of different age group and all parameters as specified were documented. It was ensure that none of the controls had history of anorectal disorders, diarrhoea or constipation. The patient group consisted of 50 children with constipation, 10 children with colostomy (histologically proved Hirschsprung's disease).

A detailed history was taken for all patients who presented with constipation, abdominal distension, failure to thrive, vomiting, and diarrhoea including family and sibling history. Main stress was laid on the passage of meconium at birth and subsequent history and severity of constipation. Total period of constipation and subsequent management 
was noted (Laxatives, stool softeners, suppositories, enemas and diet). Episodes of spurious diarrhoea and bouts of enterocolitis, fecal soiling and any previous record of documented septicaemia.In past history stress was laid on antenatal, post natal, duration of pregnancy, developmental milestones.

\section{Clinical examination:-}

General Examination of each patient was done to pick up any sign of mental retardation, endocrine imbalance.neurological status, nutritional status, failure to thrive, texture of skin, signs of hypoproteinemia, signs of septicemia and toxic megacolon.

\section{Abdominal examination:-}

Shape of the abdomen, visible peristalsis ascites, palpable bowel loops, indentable fecal masses, any associated hepatomegaly.If colostomy was already performed site, condition and management of the same was noted.

\section{Examination of perineum and rectum:-}

Position of anus and any abnormality-(anal stenosis anteriorly placed anus), fecal soiling, perianal excoriation, anal fissures, sentinel piles. Digital rectal examination for rectal gripping, tone, faecolomas and pile masses.

Investigations:-

Patients were subjected to baseline investigations which included Haemogram, Urine examination, Kidney function tests, Liver function tests,

\section{Abdominal radiograph:-}

Standing AP/lateral films were taken in each child.

\section{Barium enema:-}

It was important that the child should not have rectal irrigation or a per rectal examination before the barium enema. A catheter was inserted gently within the anus under fluoroscopic control and a small amount of barium was run in carefully. The contrast might be seen to flow into unexpanded segment and the into a cone shaped area and finally into a megacolon both A.P and lateral films should be taken. If initial films were inconclusive, delayed films 24,36 , 48 and even 72 hours can be taken.

\section{Rectal Biopsy:-}

In the early days full thickness rectal biopsy was the standard method of obtaining tissue samples for histological diagnosis, however, due to increased chances of bleeding, scarring and need of general anesthesia for all such procedures the need for submucosal biopsy was felt.

Rectal biopsy should be taken at $2 \mathrm{~cm}$ above the pectinate line as a normal area of hypogangliosis ranging from 0.3 $1.7 \mathrm{~cm}$ exists even in normal babies. If the pathologists find a stratified squamous epithelium in the specimen, then this suggests that the biopsy has been taken in normal area of hypogangliosis and a higher biopsy is warrented.Rectal biopsies should. be examined both for the presence of ganglion cells and for presence of hypertrophied nerve bundles having high acetyl cholinesterase activity.

Once the diagnosis of Hirschsprung's disease was established by anorectalmanometry, barium enema and histology, patients were subjected to colostomy. After the child had gained weight definitive surgery was performed and colostomy was closed 3 to 6 months hence. Patients were discharged and were followed up in the outpatient department.

\section{Equipment:-}

Portable Table Top AnorectalManometry System. The equipment we are using. is microprocessor based Portable AnorectalManometry System. A comprehensive platform for performing all routine AnorectalManometry investigations. Biomedical A.I.I.M.S and Department of Electronics I.I.T Delhi have developed it jointly, Manufactured by Ready Stock Mede equip (India) Pvt.Ltd. and has undergone extensive clinical trials. [Fig 1 \& Fig $2]$ 


\section{Procedure:-}

1. Informed consent was obtained from the parents of the patients, after the nature of the procedure had been fully explained in their own language.

2. The procedure necessitated a completely relaxed state for which child's confidence and cooperation was essential. Great care was taken to keep the child quiet and calm throughout the procedure. Help of an assistant was sought for young and uncooperative patients. Few patients were sedated with oral Promethazine $(0.5 \mathrm{mg} /$ $\mathrm{kg})$.

3. Each patient was given a normal saline cleansing or enema 4 hours before the procedure and no restriction on the diet was advised on patients who were on orals

4. Positioning the patient was important. Neonates and infants were kept in supine position or in the mother's lap. Older children were kept in left lateral position during the procedure.

5. Digital rectal examination was done in all the patients prior to the procedure for the subjective assessment of the tone of the sphincter,

6. Rectal dilatation, and faecalomas if present and for any structural abnormality. Also to appreciate classical gripping if present.

7. The instrument was supported at the level of the anus as this ensures that there is no pressure difference between the anal canal and the transducer and if for unavoidable reasons if it was placed at lower level, the difference in the height was subtracted from the recorded pressure.

8. Before every procedure the system box was examined, which is a rectangular container with an uncovered area at the top left hand position (a). The pressure transducer and part of the barrel of the perfusion syringe are visible here. It consists of two parts. The front portion houses the electronics (al) and the smaller pump (b). On the right side of the system box are two switches $(\mathrm{c}, \mathrm{d})$ and the receptacle for the power cord (e).

9. Power cord supplied with the machine is connected to the receptacle and the other to the mains. Power switch towards the front was put on the "ON" position. Digits appeared on the LCD panel (h), which is at the front of the system box; another window displays the message "RDY" indicating that the system is ready for use.

10. At the back of the System Box is the pump house and if the syringe was not full, it was loaded by turning the stopcock to the syringe inflow position removing the coupling pin, and rotating the lead screw until the plunger was at its posterior most position. Then coupling pin was reinserted and stop cock turned to the syringe outflow position and pump motor switched on the perfuse.

11. Calibration of the instrument prior to every manometric evaluation was done. This could be done very simply by connecting the perfusion catheter to the transducer and filling the catheter with the perfusate, either by running the pump or by infusing distilled water into the system through the side port of the three way stop cock until the water exits from the recording port of the catheter. The perfusion catheter is then raised vertically and the reading on the front panel display observed. It should read $100 \mathrm{~cm} \mathrm{H} 2 \mathrm{o}$, since the perfusion catheter is exactly $100 \mathrm{~cm}$ long and if does not, alterations will have to be made.

12. After lubricating the tip of the pressure catheter with xylocaine jelly $2 \%$, it was introduced into the rectum about 6 to 10 centimeters from the anal verge to ensure that the pressure probe was lying in the rectum and the resting rectal pressure was recorded which ranged usually between $4-10 \mathrm{cms}$ of water in normal children.

13. Pressure catheter was withdrawn at increments of $5 \mathrm{~mm}$ and time was allowed for the pressure reading to stabilize at that position, by this station pull through technique two high pressure zones were traced and were marked on the catheter, the first at the level of the internal sphincter and second at the external sphincter and pressure tracings recorded.

14. Functional length of the anal canal was measured between the two high pressure zones marked on the catheterto ensure that the rectal balloon was placed in the right position and did not slip in advertently into the anal canal during the procedure. Rectal balloons were made from the fingers of the surgical gloves sizes 6, 6.5, 7, and 7.5. and mounting them on the polyethylene tubing. Rectal balloon was placed in the position with the help of xylocaine jelly $2 \%$. To ensure that the balloon lay unfolded, it was inflated with $5 \mathrm{~m} 1$ of air, which was evacuated immediately. An assistant held the balloon tubing in position during the examination. The pressure catheter was reintroduced and recto anal pressure profile again obtained by station pull through technique. The pressure catheter was placed in the anal canal in the area with the highest anal resting zone. This region was used to study the reflex relaxation of the internal sphincter in response to rectal distension. The rectal balloon was kept inflated for 10-15 seconds. The volume of air introduced into the balloon depended on the patient's age, balloon size and the amount of rectal dilatation, which was assessed by digital rectal examination. The same method was used in all the patients.

15. The volume of air when the balloon was inflated, resulting in a passive movement of the syringe plunger, which was felt to indicate rectal distension resistance. This air volume was recorded in each patient as the volume 
required initiating an internal anal sphincter response. Distension of rectal balloon was performed by means of progressive volume increases by $5 \mathrm{~m} 1$ ration.

16. A positive RAIR (Rectoanal inhibitory reflex) is at least $50 \%$ fall in the internal sphincter pressure on inflation of the rectal balloon. RAIR (Rectoanal inhibitory reflex) was documented as positive only when three consecutive studies were consistently same. In unequivocal response repeated tests were done before documenting RAIR (Rectoanal inhibitory reflex) as positive or negative. In Hirschsprung's disease RAIR (Rectoanal inhibitory reflex) is negative. Figure 3,4,5

17. All the parameters were recorded, tabulated, plotted and printed using (Microsoft Excel Spread Sheet) on personal computer (Intel Pentium $600 \mathrm{MHz}$ ) and $840 \mathrm{C}$ (Hewlett Packard 840C Color Printer).

\section{Results:-}

The study of AnorectalManometry in the diagnosis of Hirschsprung's disease a case control study was conducted in the Department of Pediatric Surgery, Sher-I-Kashmir Institute of Medical Sciences, Soura, and Srinager. The study was carried out with effect from Dec. 99 to June 2002.The overall specificity of the test was compared with the usual modalities for the diagnosis of Hirschsprung's disease already being routinely carried out e.g. barium enema,histopathological examination. At the very outset a control study was carried out in 50 normal children to assess normal pressure profile in various age groups.

In our study we had 30 (50\%) patients with proven Hirschsprung's disease. The Rectoanal inhibitory reflex (RAIR) was true positive in 25 patients and 3 were false negative and 2 were false positive for Hirschsprung's disease. In this study 30 patients underwent barium enema plus anorectalmanometry plus rectal biopsy. Final diagnosis of Hirschsprung's disease was made in all the patients after they underwent anorectalmanometry and rectal biopsy. Age of the presentation of symptoms ranged from immediately after birth to 13 years. Majority of the cases $14(46.6 \%)$ presented between $1-5$ years. $66.6 \%$ of our patients were from rural areas. Majority of the population in Jammu and Kashmir State is from rural area. More than one symptom was present in majority of cases Delayed passage of meconium was the main presenting symptom in neonates. 3 neonates who were premature had history of delayed passage of meconium who improved on treatment and passed normal stool thereafter.Few neonates presented with features of enterocolitis/septicemia were managed in NICCU and they did well. No history of delayed passage of meconium was available in age group 5-10 years and 10-13 years. Constipation was graded mild who moved bowels every 2 days without enema or laxatives. Moderate moved bowels every 3rd day with the help of laxatives. Severe patients had palpable fecal mass, abdominal distention and palpable bowel loops. Abdominal distention was present in those patients only who were either in obstruction or had history of neglected constipation and were not getting regular enemas. No premedication was used in neonates and infants. More than one finding was present in majority of cases. Majority of the neonates had explosive discharge of liquid stool on per rectal examination which is a diagnostic feature of Hirschsprung's disease.

Faecolomas were felt per rectum in older children who were not getting regular enemas, suppositories and stool softeners. They needed to be evacuated and rectum cleansed before subjecting to anorectalmanometry. Only one patient who was 13 years old had palpable faecal masses in left iliac fossa and needed manual evacuation plus retention enemas for five days before we could do manometry .The distribution of the normal (Control) according to age, gender was done. Controls were studied for resting rectal pressure, mean resting anal canal pressure, rectoanal inhibitory reflex, functional anal canal length and the lag time which is the time lapse from the onset of rectal distension to the onset of sphincter relaxation. Volume of air used to elicit a rectoanal inhibitory reflex has been summarized in detail. In all normal controls typical pressure decrease was observed in the internal anal sphincter after air insufflations into the rectal balloon. RRP: resting rectal pressure MAP: mean anal canal pressure, Acl: anal canal length, LT: lag time

\section{Discussion:-}

Disorders of defecation are common complaints among pediatric patients and often need one or more procedures to differentiate them from aganglionicmegacolon (Hirschsprung's disease). Such procedures include barium enema, anorectalmanometry and histopathological examination to look for absence of ganglion cells and hypertrophic nerve bundles, any abnormal ganglionosis as in neuronal intestinal dysplasia which can be differentiated by immuno histochemisty e.g. Ache staining. 
Distension of the rectum normally results in the inhibition of the internal and sphincter .Gowers in 1877 was the first to report this finding. He stated that as rectal pressure increased the internal anal sphincter relaxed. This finding was also confirmed by Schuster who in 1963 used a balloon system to test anal sphincter response. In 1964, the absence of internal anal sphincter response in aganglionicmegacolon was also repeated by Callaghan and Nixon. The use of anorectalmanometry to differentiate functional from aganglionicmegacolon has evolved from their findings.

Equipment used in performing an Anorectal manometric study.

Probes:- The following types of probes are currently in use by different investigators:

1. Stiff metal hollow probes

2. Soft plastic hollow catheters

3. Dacron woven with miniature gauge located at tip so called

Digital rectal examination before every study for subjective assessment of the tone of the sphincter, rectal dilatation, faecolomas if any and for any structural abnormality and also to assess the preparation of the anorectum for performing anorectalmanometry \& judge the amount of distension that will be needed to stimulate the rectal wall in given patient and no further distension of the rectal balloon with air should be done once there is passive movement of the syringe plunger, as this may make the child uncooperative.

The balloon for rectal distension should be placed in the lower half of the rectum, which is the sensory receptor area.

The probe must be positioned properly and remains there during the whole procedure because change in the position of the probe may make it to lie in a low pressure zone and thus give erroneous results about RAIR. (Rectoanal inhibitory reflex)

Functional length of the anal canal should be measured in all the patients before the start of the procedure, a wrong length of the canal if measured will lead to placement of rectal balloon at improper site and thus rectal distension at wrong site. In all the 50 normal children who had no problems with defecation, distension of the rectum produced a decrease in the intra luminal pressure of the anal canal. The resting rectal pressures ranged from $5-11 \mathrm{~cm} \mathrm{H} 2 \mathrm{O}$ and the mean anal canal pressure ranged from 14-54 $\mathrm{cm} \mathrm{H} 2 \mathrm{O}$. The anorectal pressure studied in the patient group was similar to the pressure studied in the control group of the corresponding age group. Mishalaney concluded from the first International Symposium of Anorectal manometry in 1988 at Taipei, Taiwan (4) that it is impossible to standardize the large variety of manometric equipment used throughout the world and it is recommended that investigators should indicate their range of baseline pressure values in normal healthy individuals.In our series children with Hirschsprung's disease the resting rectal pressure ranged from $4-14 \mathrm{~cm} \mathrm{H} 2 \mathrm{O}$ and the mean anal canal pressure ranged from 10-48 cm H2O. Akira Nagasaki (5) in his study of 22 children with Hirschsprung's disease demonstrated average resting rectal pressure $11.4 \mathrm{~cm} \mathrm{H} 2 \mathrm{o}$ in the rectum and $18.8 \mathrm{~cm} \mathrm{H} 2 \mathrm{o}$ in the anal canal. The length of the anal canal was similar throughout the age range studied. In our series the functional anal canal length ranged from 1.00- $3.5 \mathrm{~cm}$. Shinji Tamate (6) in his 60 apparently healthy subjects demonstrated and canal length of $1.00-2.00 \mathrm{~cm}$, The length of the anal canal was important in performing the anorectum pressure profile because that would guide us for the placement of the rectal balloon in the rectum and would avoid achieving false negative results for Hirschsprung's disease. The time onset from of rectal distension to onset of sphincter relaxation was average 1.75 seconds in our series. Lag time was prolonged in premature neonates and could be attributed to the immature ganglion cells in these subjects. The pressures which were recorded in the anal canal in the resting phase were pressures developed by the internal anal sphincter. Consequently pressures recorded at this level reflect the activity of this sphincter. An irritated crying child puts his external anal sphincter into contraction irregularly, thereby influencing anal canal pressure, which no longer reflects internal anal sphincter activity alone. It is therefore essential that the child us not crying during the examination. In all the normal children who had no problems with defecation, distension of the rectum produced a decrease in the intraluminal pressure of the anal canal by $50 \%$ of the mean resting anal canal pressure and in children with Hirschsprung's disease. The intraluminal pressure of the anal canal rose and in few patients remained same when the rectum was distended. The results are consistent with previous reports by Akira Nagasaki(5). In response to rectal distension in control subjects, mean anal canal pressure falls due to relaxation of the internal anal sphincter, a volume of 5-60 ml was found sufficient in age group from0-13 years. After the distention the anal pressure begins to rise that is the internal sphincter increased its tone, while the rectum was still distended. Consequently if the maximal fall of anal pressure occurs immediately upon inflation of the rectal balloon, or if anal pressure does not begin to rise before the balloon is evacuated, the response should be interpreted not as a normal relaxation of the internal sphincter but as an artifact. This might be the case if the rectal 
balloon is not properly in situ, but lies folded beside the pressure probe. In our series the accuracy of anorectalmanometry has been $40 \%$ in the neonates. The diagnostic uncertainty in the neonate is thought to be the result of physiologic immaturity of the anorectal function, relative short anal canal which may result in loss of contact of the anal probe with high pressure zone during examination. Rosenberg and Meunier $\mathrm{P}(6)$ found that technical difficulties are greatest in the neonate, and doubts about the accuracy of Anorectalmanometry in the neonate have been reported In our series 20(33.3\%)of patients were diagnosed as suffering from idiopathic constipation. No false positive or false negative results were obtained. Diagnosis of idiopathic constipation in these patients was justified by persistent success of medical treatment at regular follow ups which consisted of change in dietary habits by increasing the fiber content, stool softeners, toilet training, suppositories liquid lactulose and maintaining stool charts. Anorectalmanometry was correctly diagnostic in all the patients by showing presence of RAIR (Rectoanal inhibitory reflex) Barium enema studies in patients with idiopathic constipation did not show any transition zone nor any spastic segment but $80 \%$ of patients had dilated large bowel and only 5\% patients had retained barium beyond 48 hours. Lafranchi observed that in patients with idiopathic constipation $75 \%$ of his patients had dilated bowel on barium enema. In our series we had two false positive, the absence of rectoanal inhibitory reflex without aganglionosis. Immaturity of the ganglion cells should be first cause which should be thought of Tobon and Ustach (7) examined 13 patients with Hirschsprung's disease and 71 without it, and no false positive or negative results were found. Emir $\mathrm{H}$ in his study of 36 newborn with rectal biopsies, if the results were compared to anorectalmanometry studies, there were one false positive and two false negative results. Some children had an abnormally high threshold (over $80 \mathrm{~m} 1$ for rectal distension) for the rectoanal inhibitory reflex. Due to technical problems, perfused catheters obtain small fields of pressure measurements and a small displacement of the probe could be responsible for missing the relaxation zone. In chronic constipatorsfaecolomas if present in the rectum, which would not allow a correct distension of the rectal wall an enema before the examination would certainly eliminate such a cause of error. In our series we had three false negative of Hirschsprung's disease, the finding of a rectoanal inhibitory reflex in patients with histology proven aganglionosis and can be attributed to artifacts any i.e. movement of the probe, relaxation of the external sphincter, passing of faeces, too low placement of rectal balloon so that on inflation of the balloon the and canal dilates and thus producing a reflex. Meunier et al(8) in a study of 38 neonates from 3 to 31 days of age and including seven premature infants, found the manometric diagnosis to be wrong in $(26.4 \%)$. There were four false- positive and six false-negative results. There percentage of error was $71.4 \%$ in the premature infants. Anorectalmanometry performed on patients who were already diagnosed as cases of Hirschsprung's disease revealed absence of reflex and the mean resting rectal pressures and the mean anal canal pressures were almost similar to control subjects of corresponding age group and the diagnostic accuracy in this group was $100 \%$. Our technique of performing anorectalmanometry is a simple, noninvasive well tolerated and has proved a useful tool for screening pediatric patients with chronic constipation. Patients who were positive for Hirschsprung's disease on anorectalmanometry were subjected to barium enema to estimate the length of the involved segment and to compare the accuracy of anorectalmanometry and barium enema in the diagnosis of Hirschsprung's disease. Classic barium enema finding was demonstrated in $73.3 \%$ of our patient's. Flow of barium from the undilated rectum through a cone shaped transition zone into the dilated colon and was best appreciated by a lateral film. However in half of the newborns and infants the colon was not appreciably dilated therefore typical cone was not seen. The accuracy of barium enema in neonates was improved by taking films 24 hours later and in few even up to 72 hours when it showed retained barium. Janet R. Reid(9) from her study of 48 patients concluded that the barium enema had a sensitivity and positive predictive value of $83 \%$ and specificity and negative predictive value of $98 \%$ for diagnosing Hirschsprung's disease. Barium enema is a diagnostic tool but its limitation must be appreciated. However barium enema performed by an experienced radiologist using careful technique should give better results. Using dilute barium (25\%weight by volume in dilution) and keeping the can at the height of 4 feet, injecting slowly under fluoroscopic control and using air as contrast can give outline of the anorectum better and taking early lateral and oblique views to avoid overlapping of sigmoid colon over the rectal area. Rectal washouts or even digital examination should be avoided prior to examination, because this they distort the transition zone. In patients in whom anorectalmanometry and barium enema was suggestive of Hirschsprung's disease, they were subjected to rectal biopsy to confirm the diagnosis. A side biting, long, laryngeal forceps was used to obtain two superficial mucosal specimens taken $4 \mathrm{~cm}$ above the anal verge. Specimen was sent for routine H.E staining and if the normal ganglion cells were seen then no further work up was required. These children were put on medical management for constipation and kept under follow up. Patients who had equivocal results were subjected to immunohistochemistry studies such as Ache staining and if on Ache staining the mucosa was intense with an abundance of nerve fibers and ganglion cells were absent then diagnosis of Hirschsprung's was made. Simple suction biopsies are less traumatic and can be done as an O.P.D. procedure with sedation. Disadvantage being at times tissue is inadequate therefore its histology may be in conclusive. The diagnostic accuracy of 
anorectalmanometry in our series was $83.3 \%$, for routine histology exarnination $94.2 \%$ and acetyl cholinesterase histochemistry 100\%.Irecmichi(10) studied 309 constipated infants and children who underwent rectal manometry, routine histological examination. They achieved $99 \%$ diagnostic accuracy in acetylcholine histochemistry and $61 \%$ in H. E staining. Finally we conclude that children referred to our Institute with complaints of constipation the following protocol should be followed. It consists of a detailed clinical history, physical examination, anorectalmanometry, barium enema, rectal biopsy and routine H.E staining and if inconclusive Ache staining. Our technique of anorectalmanometry is simple, non invasive and well tolerated and has proved useful for screening pediatric patients with chronic constipation although we have encountered no complications but should be avoided in neonates with necrotizing enterocolitis because of mucosal edema and friable rectal wall which make the chances of perforation high. Anorectalmanometry when done properly eliminates the need of barium enema for diagnostic accuracy of Hirschsprung's disease. The latter should be done only when the clinical profile does not match with the manometric findings e.g. no definite response with medical management. However histopathological examination is a must before subjecting the patient for definitive treatment for Hirschsprung's disease.

\section{Conclusion:-}

The study of Anorectal manometry in the diagnosis of Hirschsprung's disease a case control study was conducted in the Department of Paediatric Surgery, Sher-I-Kashmir Institute of Medical Sciences from December 99 to June 2002. Control subjects consisted of 50 children whose ages ranged from 1 day to 13 years; none of the controls had any history of defecation disorder. All the controls had rectoanal inhibitory reflex present at the time of examination. Resting rectal pressures recorded in them ranged from 5-11 cmH,0 and the Mean anal canal pressure ranged from 14$54 \mathrm{~cm} \mathrm{H} 2 \mathrm{O}$.Functional anal canal length ranged from 1.0-3.5cms.In our series $50 \%$ of patients were diagnosed to be suffering from Hirschsprung's disease and $33.3 \%$ were diagnosed to be suffering from idiopathic constipation. Youngest patient was 10 days old who presented with necrotizing enterocolitis of Hirschsprung's disease and the oldest presented as a case of neglected chronic constipation in intestinal obstruction. Anorectal pressure profile did not differ much between the control group and the patient group with average resting rectal pressure ranging from 4$14 \mathrm{cmH} 20$ and mean anal canal pressure ranging from 10-48 cmH20.The length of the anal canal averaged 1.0-3.0 cms. There were 3 false negatives and 2 false positives for Hirschsprung's disease on anorectal manometry with overall diagnostic accuracy of $83.3 \%$. Anorectal manometry should be recommended as an initial screening test of patients with chronic constipation because it is safe, reliable, and inexpensive, can be repeated, least invasive, has a physiologic basis and can be done safely in the neonates however it should be supplemented with proper histopathological examination and a good contrast enema before instituting definitive treatment for Hirschsprung's disease.

\section{References:-}

1. Arvin I.Philippart.Hirschsprung'sdisease.Aschcrofi Holder. Pediatric Surgery 2nd Edition W.B.Saunder Company 1993

2. Rosenberg Allan J.and Vela A. Richard .A new simplified Technique for Pediatric AnorectalManometry. Pediatrics 1983; 71(2):240-245.

3. Henry G.Mishalany and Morton M.Wooley. Postoperative Functional and Manometric Evaluation of Patients with Hirschsprung's disease. Journal of Pediatric Surgery, 1987; 22(5): 443- 446.

4. Molnar D. Taitz L.S, Urwin O.M. AnorectalManometry- Results in Defecation Disorders. Arch Dis Child 1983; 58(4):257-261.

5. Akira Nagasaki. Postoperative Sequential AnorectalManometric Study of Children with Hirschsprung's disease. Journal of Pediatric Surgery 1980; 15(5)615-619.

6. SatishS.PatelS.How useful areManometric tests of Anorectal function in the management of Defecation Disorders? Am J Gastroenterol 1997; 92(3):469-475.

7. Thomas J.Ustah, Fabio Tobon, Marvin M Schuster. Simplified Method for Diagnosis of Hirschsprung's disease. Arch Dis child 1969; 44:694-697.

8. Vera A. Loening-baucke.AnorectalManometry: Experiance With strain Gauge Pressure Transducers for the Diagnosis of Hirschsprung's disease. Journal of Pediatric Surgery.1983; 18(5).

9. Janet R.Reid, Carlo Buonomo, Claudio Moreira Harold Kozakevich, Samuel J.Nurko. The barium enema in constipation: Comparison with rectal manometry and biopsy to exclude Hirschsprung's disease after the neonatal period. Pediatric Radiol 2000; 30:681-684.

10. Iremichilkawa, Samuel H.Kim, W.HardyHendren, Patricia K Donahoe.Acetylcholinesterase and Manometry in the Diagnosis of the Constipated child. Arch Surg-1986; 121:435-438. 